\title{
IDENTIFICAÇÃO DE DIFERENTES ESTÁGIOS SUCESSIONAIS E IDADE DA FLORESTA: ABORDAGENS DO SENSORIAMENTO REMOTO
}

\author{
IDENTIFICATION OF DIFFERENT SUCCESSIONAL STAGES AND FOREST AGE: REMOTE \\ SENSING APPROACHES
}

IDENTIFICACIÓN DE DIFERENTES ETAPAS SUCESIONALES Y EDAD FORESTAL: ENFOQUES DE DETECCIÓN REMOTA

Elaine de Cacia de Lima Frick Doutoranda em Geografia, UFPR Brasil elaineclfrick@gmail.com 


\section{RESUMO}

Áreas florestais que são abandonadas após distúrbios sejam de ordem natural ou antrópica passam por um processo natural de regeneração da vegetação. Este processo permite a formação de florestas secundárias, as quais necessitam de estudos e monitoramento devido sua função ambiental na recuperação de áreas degradadas, no estoque de carbono e nutrientes e como reguladoras do ciclo hidrológico etc. Contudo, para entender esta dinâmica de regeneração e conhecer suas funções ambientais se faz necessário muitas vezes detectar a idade da vegetação, tornando-se uma variável importante a ser estudada. Dentre os meios para identificação dos estágios sucessionais e idade da floresta, têm-se o uso das técnicas de sensoriamento remoto (SR), para tanto o objetivo deste trabalho foi analisar e discutir estudos que empregaram tais técnicas para identificar os estágios sucessionais e idade da floresta. As etapas metodológicas realizadas foram: Fundamentação teórica; Revisão Sistemática de Literatura (RSL) com a seleção de 10 estudos; e Análise e discussões dos resultados. Os métodos empregados envolveram técnicas de classificação e modelos preditivos: Pós-classificação de fusão; Classificação Supervisionada; Feição de textura; Índices Espectrais; Modelo de Mistura Espectral (MME); Modelo de Regressão; Redes Neurais (RN); Árvores de Decisão; e Floresta Aleatória. Os estudos analisados trouxeram resultados satisfatórios de acordo com os seus objetivos, recorte espacial e técnicas empregadas, confirmando a importância da identificação dos estágios sucessionais e idade da floresta utilizando o SR na automatização do processo de aquisição de informações.

PALAVRAS-CHAVE: Dados de detecção remota. Métodos de classificação. Modelos preditivos.

\section{ABSTRACT}

Forest areas that are abandoned after natural or anthropogenic disturbances undergo a natural process of vegetation regeneration. This process allows the formation of secondary forests, which need studies and monitoring due to their environmental function in the recovery of degraded areas, carbon and nutrient stock and as regulators of the hydrological cycle. However, to understand this regeneration dynamics and to know its environmental functions, it is often necessary to detect the age of the vegetation, becoming an important variable to be studied. Among the means for identifying successional stages and forest age, remote sensing (SR) techniques have been used. Therefore, the objective of this paper was to analyze and discuss studies that employed such techniques to identify successional stages and forest age. The methodological steps performed were: Theoretical foundation; Systematic Literature Review (RSL) with the selection of 10 studies; and Analysis and discussion of results. The methods employed involved classification techniques and predictive models: Fusion post-classification; Supervised Classification; Texture feature; Spectral indices; Spectral Mixture Model (MME); Regression model; Neural Networks (RN); Decision trees; and Random Forest. The analyzed studies brought satisfactory results according to their objectives, spatial clipping and techniques employed, confirming the importance of identifying successional stages and forest age using SR in the automation of the information acquisition process.

KEYWORDS: Remote sensing data. Classification methods. Predictable models.

\section{RESÚMEN}

Las áreas forestales que se abandonan después de perturbaciones naturales o antropogénicas experimentan un proceso natural de regeneración de la vegetación. Este proceso permite la formación de bosques secundarios, que necesitan estudios y monitoreo debido a su función ambiental en la recuperación de áreas degradadas, carbono y reservas de nutrientes y como reguladores del ciclo hidrológico. Sin embargo, para comprender esta dinámica de regeneración y conocer sus funciones ambientales, a menudo es necesario detectar la edad de la vegetación, convirtiéndose en una variable importante para ser estudiada. Entre los medios para identificar las etapas sucesionales y la edad del bosque, se han utilizado técnicas de teledetección (SR). El objetivo de este trabajo fue analizar y discutir los estudios que emplearon tales técnicas para identificar las etapas sucesionales y la edad del bosque. Los pasos metodológicos realizados fueron: fundamento teórico; Revisión sistemática de la literatura (RSL) con la selección de 10 estudios; y Análisis y discusión de resultados. Los métodos empleados incluyeron técnicas de clasificación y modelos predictivos: post-clasificación de Fusion; Clasificación supervisada; Característica de textura; Índices espectrales; Modelo de mezcla espectral (MME); Modelo de regresión; Redes neuronales (RN); Árboles de decisión; y bosque al azar. Los estudios analizados arrojaron resultados satisfactorios de acuerdo con sus objetivos, recorte espacial y técnicas empleadas, confirmando la importancia de identificar etapas sucesionales y la edad del bosque utilizando SR en la automatización del proceso de adquisición de información.

PALABRAS CLAVE: Datos de teledetección. Métodos de clasificación. Modelos predictivos. 


\section{INTRODUÇÃO}

Áreas florestais que foram abandonadas após sofreram distúrbios por ações naturais ou antrópicas passam por um processo natural de regeneração da vegetação, nestes locais podem surgir processos sucessionais que dão origem às florestas secundárias (FOSBERG, 1967). Tais florestas apresentam diversos estágios sucessionais, que tendem a alcançar uma comunidade mais complexa, diversa e estável, sendo um meio de renovação das florestas tropicais (ATTANASIO et al., 2006; KAGEYAMA e CASTRO, 1989; KLEIN, 1980).

Florestas secundárias precisam ser estudadas e monitoradas, pois desempenham importante papel na recuperação do solo através da acumulação de biomassa (MORAN et al., 2000), são extremamente importantes como reserva da diversidade genética, estoque de carbono e nutrientes e reguladoras do ciclo hidrológico (VIEIRA et al., 2003), na troca de energia e no processo de sistema terrestre ecológico (PETERSON et al., 1988). Informação sobre taxonomia vegetal, sua distribuição espacial e dinâmica temporal são fundamentais para o êxito do sistema ecológico (JENSEN et al., 1999). Chazdon et al. (2009); Dent e Wright (2009) relatam que florestas secundárias podem oferecer habitats adequados às várias espécies florestais.

Jesen et al. (1999) relatam que estudos tentaram avaliar e prever a biofísica da floresta, contudo variações ocorridas durante esses estudos foram frequentemente relacionadas à idade ou maturidade da planta, tornando-se uma variável a ser estudada. Para Foody et al. (1996) a regeneração da floresta em áreas previamente desmatadas, é um sumidouro significativo de carbono, sendo a força desse sumidouro de carbono dependente da idade e da composição da floresta em regeneração.

Desta forma, a idade da vegetação permite o entendimento sobre a dinâmica do processo de regeneração, pois a distribuição de fragmentos florestais de idades distintas dentro de uma paisagem é um indicador chave do potencial para regeneração florestal de um sítio recém abandonado (CHAZDON et al., 2009). A idade é uma variável importante que pode ser de valor significativo quando se pretende modelar, por exemplo, a saúde do ecossistema dominado pela floresta (JENSEN et al., 1999).

Inúmeros estudos são efetuados com o intuito de obter o mapeamento das classes de vegetação, avaliar e monitorar áreas degradadas, verificar a restauração florestal e recuperação biológica, além de quantificar o orçamento de carbono de uma região, obtendo estimativas temporais da extensão e idade de regeneração de florestas tropicais.

Com o surgimento das imagens de Satélite, estes estudos passaram a ser realizados por meio de técnicas de Sensoriamento Remoto (SR) (HELLER e ULLIMAN, 1983; PONZONI, 2001; TRABAQUINI et al., 2007); vários autores vêm pesquisando como classificar automaticamente os estágios sucessionais da vegetação (VIEIRA et al., 2003; GALVÃO et al., 2009; AMARAL et al., 2009; LI et al., 2011; LU et al., 2012; LU et al., 2014), facilitando desta forma o processo de detecção e de análise.

O desafio dos pesquisadores ao utilizar do SR está em realizar o mapeamento preciso da floresta secundária e de descobrir sua idade, já que há dificuldades devido às semelhanças espectrais entre diferentes estágios sucessionais e variações na refletância de florestas com variação das 
trajetórias de regeneração (HELMER et al., 2000; LUCAS et al., 2000; NEEFF et al., 2006). Em relação a sua estrutura quanto mais velha a floresta tropical esta se apresenta mais diversificada do que a floresta secundária mais jovem (HELMER et al., 2009), porém o desafio está em verificar se tal diferença também é perceptível segundo análise espectral.

Grant (1987) aponta que há mudanças na clorofila das folhas e na estrutura interna durante o ciclo fenológico de uma planta, afetando significativamente seu padrão de resposta espectral, sendo possível então o mapeamento da floresta regenerada e sua idade.

Para Li et al. (2014) os sinais espectrais detectados remotamente não são uma função explícita da idade da floresta, pelo contrário, eles estão ligados a fatores estruturais relacionados à idade, como: biomassa, índice de área foliar, densidade, área e altura. Sendo assim, é possível rastrear os estágios de sucessão florestal e manter a idade de acordo com mudanças nos sinais espectrais, podendo extrair a idade da floresta usando dados detectados remotamente em uma série única ou temporal.

\section{OBJETIVO}

Este artigo teve como objetivo analisar e discutir estudos que empregaram as técnicas de SR na identificação e classificação dos estágios sucessionais e idade da floresta.

\section{METODOLOGIA}

Para realização do trabalho, seguiram-se três etapas metodológicas principais:

- A primeira consistiu na fundamentação teórica, acerca dos temas pertinentes ao objetivo (floresta secundária, estágios sucessionais e detecção da idade da vegetação por meio do $S R)$.

- A segunda etapa referiu-se à Revisão Sistemática de Literatura (RSL), adotada como um método de pesquisa que utiliza a literatura sobre determinado tema como fonte de dados, abrangendo um resumo das evidências científicas de acordo com a aplicação de métodos de busca, síntese da informação selecionada e apreciação crítica dos resultados. De acordo com Denyer e Tranfield (2009); Medeiros et al. (2014); Garza-Reyes (2015), a RSL consiste em cinco fases consecutivas: (1) formulação da questão, (2) localização dos estudos, (3) avaliação e seleção dos estudos, (4) análise e síntese, e (5) relatar e usar os resultados. Nesse sentido, a Figura 1 apresenta como a seleção dos estudos foi realizada para este trabalho, onde a questão definida referiu-se a busca de trabalhos que empregassem diferentes metodologias para identificação da sucessão vegetacional e idade da floresta, apresentando resultados e discussões por meio do uso das técnicas de SR. O resultado da RSL proporcionou obter 10 trabalhos para análise, sendo estes 9 (nove) artigos científicos e 1 (uma) dissertação, inicialmente a intenção era de analisar apenas teses e dissertações, porém houve dificuldades em encontrar teses que associavam o uso de SR na identificação da idade da vegetação, sendo necessário utilizar o método de RSL na busca de artigos científicos.

- A terceira etapa consistiu na análise e discussão dos 10 trabalhos selecionados, organizados no Quadro 1. 
Figura 1: Fluxograma da Seleção dos Estudos

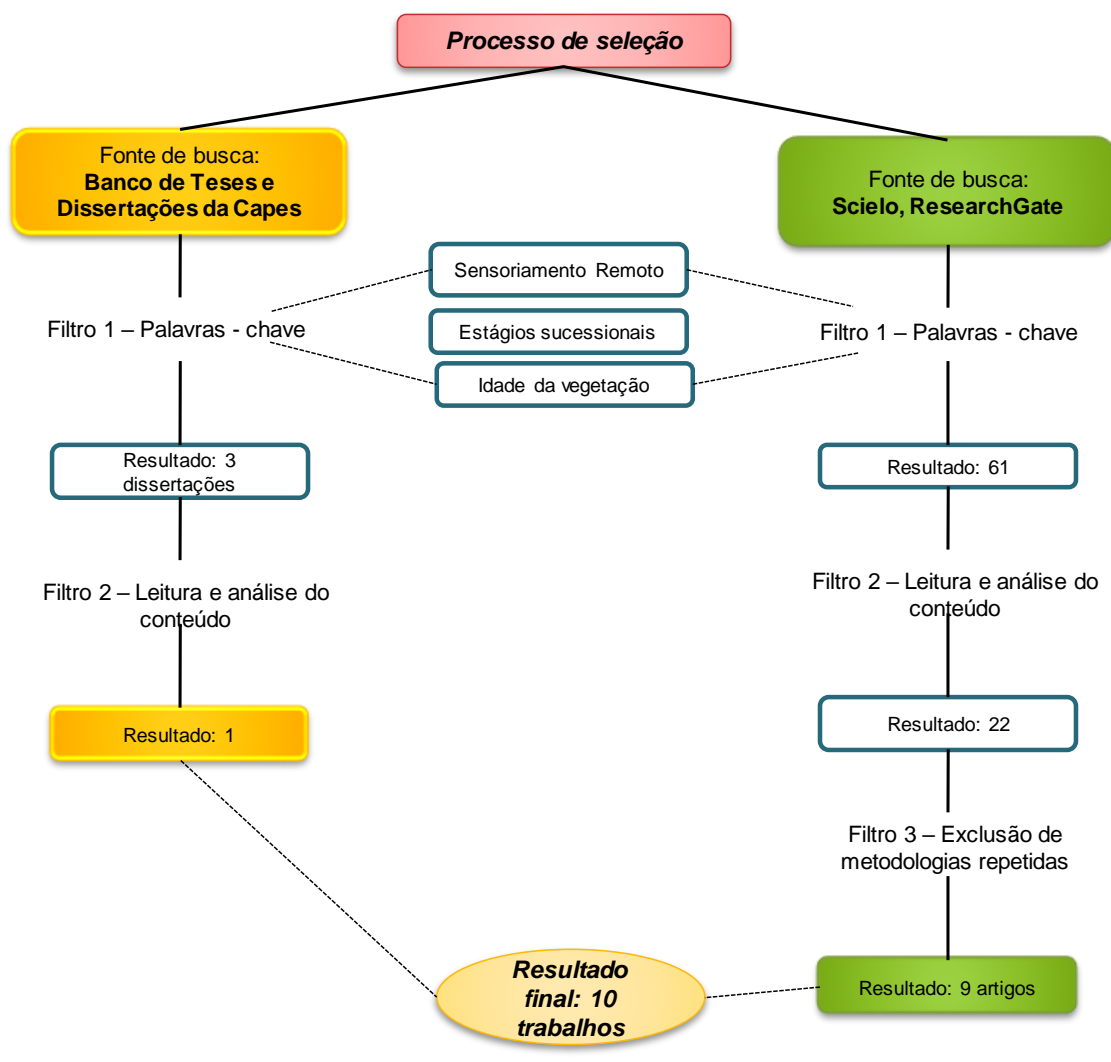

Org. a autora (2019).

Quadro 1: Estudos selecionados para análise 


\begin{tabular}{|l|l|l|}
\hline \multicolumn{1}{|c|}{ ESTUDO } & \multicolumn{1}{|c|}{ OBJETIVO } & \multicolumn{1}{|c|}{ ÁREA DE ESTUDO } \\
\hline LUCAS et al. (1993) & Estudos de carbono & Floresta Amazônica \\
\hline STEININGER (1996) & $\begin{array}{l}\text { Uso do Landsat para estimativa de área e idade } \\
\text { de rebrota da floresta secundária }\end{array}$ & Floresta Amazônica \\
\hline FOODY et al. (1996) & Identicação de sumidores de carbono terrestre & Floresta Amazônica \\
\hline KIMES et al. (1999) & Testar SPOT HRV para discriminação da floresta & Floresta Amazônica \\
\hline FRASER e LI (2002) & $\begin{array}{l}\text { Testar SPOT VGT para estimar parâmetros } \\
\text { relacionados ao incêndio }\end{array}$ & Floresta Boreal - Canadá \\
\hline KENNAWAY e HELMER (2007) & $\begin{array}{l}\text { Quantificar as idades e tipos de florestas } \\
\text { submetidas ao desenvolvimento terrestre }\end{array}$ & Porto Rico \\
\hline MAILLARD e COSTA-PEREIRA (2010) & $\begin{array}{l}\text { Estudar o processo de regeneração da vegetação } \\
\text { de cerrado }\end{array}$ & Cerrado - Minas Gerais \\
\hline NUNES (2011) & $\begin{array}{l}\text { Diferenciar classes de idades de florestas } \\
\text { secundárias usando Landsat }\end{array}$ & Floresta Amazônica \\
\hline GOMES e MAILLARD (2013) & $\begin{array}{l}\text { Modelar o processo de regeneração da } \\
\text { vegetação de cerrado }\end{array}$ & Cerrado - Minas Gerais \\
\hline CARREIRAS et al. (2017) & Recuperar a idade de florestas secundárias & Floresta Amazônica \\
\hline
\end{tabular}

Fonte: Estudos selecionados. Org. a Autora (2019).

\section{RESULTADOS}

A partir da análise dos estudos selecionados eles foram classificados conforme o tipo de dados e métodos de SR adotados na identificação dos estágios sucessionais e sua idade. Os estudos foram divididos em 2 (dois) grupos em relação ao tipo de dados: estudos que utilizaram série temporal de imagens de satélite e estudos que utilizaram imagem de data única. E para o uso dos métodos, também foram divididos em 2 (dois) grupos: os que utilizaram-se de técnicas paramétricas e os que empregaram as técnicas não paramétricas.

\subsection{TIPOS DE DADOS DE SENSORIAMENTO REMOTO UTILIZADOS}

Ressalta-se que a maioria dos estudos analisados empregaram a série temporal do Landsat (LUCAS et al., 1993; FOODY et al., 1996; STEININGER, 1996; KENNAWAY e HELMER, 2007; MAILLARD e COSTA-PEREIRA, 2010), devido à disponibilidade histórica, uma vez que os dados Landsat estão disponíveis desde 1973 permitindo o acompanhamento da trajetória do distúrbio e da regeneração da vegetação, facilitando a recuperação da idade da floresta. As séries temporais de dados de SR se apresentam mais eficazes para detectar o histórico de perturbações florestais e idade da floresta (LI et al., 2014).

Contudo, atesta-se que este fator depende do objetivo da pesquisa e se a idade da floresta é uma variável conhecida ou não. Há de se destacar também a dificuldade na maioria das vezes em obter todas as imagens de uma série histórica do Landsat devido às condições atmosféricas, onde a presença de nuvens torna-se muitas vezes um obstáculo para o reconhecimento e mapeamento da vegetação. 
No estudo de Kennaway e Helmer (2007) também foi utilizada uma série temporal de fotografias aéreas devido à indisponibilidade de imagens Landsat anterior ao ano de 1973. Kimes et al. (1999) utilizaram uma série histórica do SPOT HRV (HIGH RESOLUTION VISIBLE), para separação das classes de: floresta primária, floresta secundária e área desmatada. Fraser e Li (2002) usando uma série temporal do SPOT VGT (VEGETATION) apresentaram um método para estimar a idade da floresta (pós-queima).

Os estudos que utilizaram imagem de data única foram: dados do Landsat (NUNES, 2011), RapidEye (GOMES e MAILLARD, 2013) e Alos Palsar combinado com Landsat (CARREIRAS et al., 2017). Em Nunes (2011) foi utilizada uma única imagem Landsat (a mais recente da série temporal) para avaliar espectralmente as idades de florestas. No estudo de Gomes e Maillard (2013) as imagens do RapidEye foram utilizadas por apresentar alta resolução espacial, como forma de se verificar melhorias nas estimativas de idade da regeneração do cerrado devido os detalhes na imagem. Já o uso dos dados do Alos Palsar em Carreiras et al. (2017) foi justificado devido à cobertura de nuvens em áreas tropicais e porque dados de Radar de Abertura Sintética (SAR) tornam-se úteis ao recuperar a idade das florestas secundárias e complementar o que é fornecido por sensores ópticos.

\subsection{MÉTODOS DE SENSORIAMENTO REMOTO APLICADOS}

Os trabalhos analisados empregaram modelos preditivos e de classificação para estimar/predizer/detectar/recuperar/distinguir os estágios de sucessão e idade da vegetação. Em Lucas et al. (1993) usando pós-classificação de uma sequência multitemporal de imagens do sensor Landsat, os autores conseguiram classificar cinco estágios de regeneração da floresta secundária, juntamente com floresta primária e terras agrícolas.

Foody et al. (1996), adotaram a classificação supervisionada por meio do classificador de Máxima Verossimilhança (MAXVER) para separar as classes de idade de floresta tropical (pasto, $<2$ anos, 2-3 anos, 3-6 anos, 6-14 anos,> 14 anos) obtendo mais de 85\% de precisão da classificação usando bandas do Landsat TM 3, 4, e 5 (vermelho, infravermelho próximo e infravermelho médio respectivamente). A escolha destas regiões espectrais refere-se na melhoria da discriminação de temas de uso do solo e, sobretudo na identificação da vegetação, devido à sua alta refletância na região do infravermelho próximo, seu contraste com a região do vermelho onde ocorre a maior absorção de energia e o controle da sua curva espectral no infravermelho médio devido à presença de umidade.

Questiona-se o uso de classificadores tradicionais (a maioria de cunho paramétrico), por não obterem maior precisão na classificação devido à heterogeneidade da cobertura do solo (ALVES e SKOLE, 1996 e BRONDíZIO et al., 1996). Outro fato a ser indagado, refere-se aos classificadores tradicionais não conseguirem lidar efetivamente com o problema do pixel misto, devido à limitação na resolução espacial onde um único pixel muitas vezes contém mais de uma classe de cobertura (LU et al., 2003). 
No estudo de Nunes (2011) foram utilizados testes estatísticos para comparar imagens em refletância, imagens-fração do Modelo de Mistura Espectral (MME) (vegetação - GV, NPV, solos e sombra) e Índices espectrais (IE): índices de fração (NDFI) e vegetação (NDVI) para distinguir floresta primária da maioria das classes de idade de floresta secundária, concluindo que as florestas apresentaram diferenças significativas a partir de 20 anos de distinção entre elas, considerando sua assinatura espectral.

No MME uma relação linear é utilizada para representar a mistura espectral dos alvos em cada pixel. Assim, a resposta espectral dos pixels em qualquer comprimento de onda pode ser considerada como uma combinação linear de cada componente da mistura. Tendo como objetivo de separar cada elemento do pixel da imagem para obter uma imagem fração para cada endmember considerado (SHIMABUKURO e SMITH, 1991). As imagens fração representam o porcentual de cada endmember nas imagens originais (QUINTANO et al., 2011). Este é um dos métodos mais utilizados para resolver o problema do pixel misto em imagens.

Já os IE entre eles os índices de vegetação (IV) são aplicados para monitorar e quantificar as condições e distribuições espaciais da vegetação, utilizando-se de dados digitais de refletâncias. Os IV são obtidos das várias combinações matemáticas das refletâncias em várias regiões espectrais da radiação eletromagnética. Num primeiro momento o objetivo é utilizar os IV para condensar as informações espectrais e discriminar o que é vegetação e não vegetação (LIU, 2006).

Em Gomes e Maillard (2013) foram avaliados o potencial das imagens RapidEye (devido a alta resolução espacial) e o incremento de feições de textura para estimativa da idade e de medidas estruturais da vegetação do cerrado em diferentes subformações e estágios sucessionais. Unindo feições espectrais e texturais os autores geraram um modelo de estimativa da idade da vegetação do cerrado (0-37 anos) com coeficiente de determinação de $80,05 \%$ e coeficiente de determinação ajustado de $75,3 \%$.

Reafirmando os resultados obtidos pelo estudo acima, a textura é uma das técnicas de análise de imagem a ser utilizada para a extração de informação estrutural de florestas de uma imagem digital (WULDER, 1998).

Destacam-se os estudos em que os autores empregaram os algoritmos não-paramétricos: Modelo de Regressão (MAILLARD e COSTA-PEREIRA, 2010); Rede Neural (RN) (STEININGER, 1996; KIMES et al., 1999; FRASER e LI, 2002); Árvore de decisão (KENNAWAY e HELMER, 2007); e Floresta Aleatória (CARREIRAS et al., 2017).

Tais técnicas não paramétricas tornaram-se proeminentes na literatura para eliminar a limitação de suposições estatísticas paramétricas (HANSEN et al., 1996; FRIEDL e BRODLEY, 1997; LAWRENCE e WRIGHT, 2001; PAL e MATHER, 2003; ROGAN et al., 2003; KRISHNASWAMY et al., 2004). Melhorando assim a modelagem preditiva de parâmetros biofísicos do povoamento florestal, quando utilizadas adequadamente (JENSEN, 1999).

As RN muitas vezes são técnicas empregadas devido à dificuldade em detectar a idade florestal após o abandono e seu sinal espectral, já que isto depende de vários fatores, como: estrutura da vegetação, propriedades ópticas, iluminação e topografia, também devido à complexidade nas formas de desmatamento, o uso da terra antes da regeneração da floresta, as propriedades do solo e outras características de qualidade do sítio. Por conta de todas estas variáveis, o uso 
de um modelo físico como a RN possibilita a relação complexa das variáveis obtendo no fim maior precisão (KIMES et al., 1999).

No estudo de Steininger (1996) utilizando quatro imagens Landsat com o objetivo de estimar idade, área e mudanças em florestas secundárias em Manaus e ao usar uma RN ele constatou que as assinaturas espectrais eram distintas entre floresta tropical primária e secundária até aproximadamente 14 anos, no entanto descobriu que a floresta secundária mais velha passa a se misturar espectralmente com a floresta primária.

Kimes et al. (1999) também utilizaram RN conseguindo separar: floresta primária, floresta secundária (estimada em até > 9 anos) e pixels desmatados, obtendo uma precisão de $95,2 \%$.

Fraser e Li (2002) com um modelo de RN desenvolvido usando métricas temporais, estimaram com um erro RMS de 7 anos a idade da floresta pós-queima. Concluíram que o instrumento VGT foi eficaz para mapear grandes queimadas boreais no final de uma estação de fogo e aproximar a idade de regeneração de queimadas com menos de 30 anos de idade.

Apesar dos 3 (três) trabalhos acima terem utilizados as RN, cada um traçou objetivos e alcançou resultados específicos às suas aplicações.

Em Kennaway e Helmer (2007) foram empregadas as Árvores de decisão para descobrirem quais florestas foram desmatadas para o desenvolvimento da terra, como resultado eles identificaram de que a maioria eram florestas jovens (1-13 anos) contabilizando $55 \%$ da área, tal resultado serviu para avaliar as conseqüências da supressão vegetacional na área de estudo.

O uso de árvores de decisão se dá por dois motivos principais: primeiro, os classificadores de árvore de decisão acomodam classes espectralmente heterogêneas, tanto o tempo como os dados de treinamento representam a variabilidade espectral da classe, as árvores de decisão podem acomodar parte da variabilidade espectral da classe que vem das diferentes datas de imagens que compõe um mosaico, por exemplo, (FRIEDL e BRODLEY, 1997; WOODCOCK et al., 2001); e em segundo lugar, as árvores de decisão podem lidar com muitas variáveis preditoras contínuas, separando tipos de florestas espectralmente similares das variáveis como: chuva ou geologia (STRAHLER, 1981; SKIDMORE, 1989).

No estudo de Maillard e Costa-Pereira (2010) eles apontaram como resultado a criação de um modelo de regressão múltipla da idade da regeneração do cerrado gerado a partir das bandas da imagem Landsat de 2007 conseguindo explicar 61\% da variância observada. Os resultados mostraram que dados espectrais podem ser usados para estimar a regeneração de idade até 30 anos com uma precisão média de 3-4 anos.

Em Carreiras et al. (2017) usando a Floresta Aleatória eles conseguiram mapear as florestas maduras, floresta secundária e área não-florestal, com uma precisão global de 95-96\% no período 2007-2010. Este estudo obteve maior precisão perante os demais na discriminação entre o tipo de floresta e área não florestal, tal fato associa-se a combinação entre dados do Landsat e Alos Palsar além do uso do algoritmo de aprendizagem de máquina (Floresta Aleatória).

O algoritmo de florestas aleatórias (RF) é construído como um conjunto de árvores de decisão binária, com apenas dois parâmetros precisando ser ajustados: o número de árvores em cada modelo de RF e o número de preditores selecionados aleatoriamente para serem usados em cada nó de decisão (BREIMAN, 2001). 
A Floresta Amazônica foi escolhida como recorte espacial na maioria dos estudos analisados (totalizando seis), afirmando a importância deste bioma para pesquisadores de diversos locais, destacando a preocupação em relação à degradação sofrida nesta região e a substituição das florestas primárias na maioria das vezes por extensas áreas de pastagem e monocultura. Tais estudos reforçam a necessidade de pesquisas nesta região, pois com a supressão da floresta primária e mais tarde com o abandono das áreas ocupadas por outros usos, a floresta secundária surge num processo de regeneração, como já embasado anteriormente, tornando o seu estudo fundamental para a recuperação de áreas degradadas e para entender o quanto estas florestas podem auxiliar no estoque de carbono. Sendo apontado por alguns autores que a floresta secundária sequestra carbono mais rapidamente do que florestas primárias ao longo de muitas décadas (UHL et al.,1988; DIXON et al., 1994; FEARNSIDE e GUIMARÃES, 1996) evidenciando a necessidade de estudos que tratem desta temática e que tragam resultados científicos em relação a esta afirmativa.

Com os resultados e análises dos estudos selecionados, verificou-se que o SR por meio de seus produtos e métodos atendeu aos objetivos dos pesquisadores, uma vez, que foi possível a identificação e discriminação das classes de estágios sucessionais e obtenção da idade da floresta. Segundo Steininger (1996) utilizando estas técnicas de SR é possível estimar as características das florestas, medidas pela refletância espectral e índices resultantes da interação entre a superfície da terra e o sensor.

Entretanto, faz-se necessário citar Cohen et al. (1995) onde apontam que é preciso sempre ter em mente que a medição direta e invasiva do valor de idade da vegetação não é possível, o que pode-se medir são manifestações que são sua consequência, destacando-se que pode ser único para cada localidade, devido por exemplo a história do distúrbio. Neste trabalho, foi possível constatar que cada pesquisador utilizou distintos dados e técnicas de SR em virtude de seus objetivos, obtendo resultados específicos diante de seu recorte espacial, da trajetória de distúrbio e do processo de regeneração da floresta, ficando claro não existir uma rotina única e absoluta a ser aplicada em áreas florestais.

\subsection{INVESTIGAÇÕES FUTURAS}

Espera-se que em pesquisas futuras relacionadas aos avanços do SR na identificação dos estágios sucessionais e idade da floresta, sejam realizadas:

- Um maior número de pesquisas sobre outras regiões no Brasil, como por exemplo, na Floresta Atlântica onde o relevo é mais heterogêneo apresentando áreas com maiores declividades, já que a maioria dos estudos obtidos refere-se à Floresta Amazônica. Nos locais com maiores inclinações na Floresta Atlântica ocorrem com relativa frequência os eventos de movimentos de massa, onde os estudos voltados ao processo de regeneração da vegetação para reconstituição paisagística e recuperação de área tornam-se necessários;

- Incentivo para que um número maior de pesquisas faça uso combinado de diferentes dados de SR, melhorando a capacidade de extrair a informação, assim como o seu processamento; 
- Maior acessibilidade as imagens orbitais de alta e altíssima resolução espacial, para que pesquisadores possam testar suas aplicações no processo de identificação da vegetação em diversos estágios de regeneração e obtenção da idade, explorando sua capacidade de apresentar maiores detalhes;

- Acréscimo de pesquisas que explorem o uso de sensores que apresentam "novas" regiões espectrais (como por exemplo, as bandas do amarelo e borda do vermelho), avaliando se a resposta espectral nestas regiões permite maior discriminação entre os estágios sucessionais e as diferentes idades;

- $\quad$ Aumento da investigação sobre o potencial dos dados SAR para auxiliar na estimativa da idade da vegetação;

- Que o mapeamento de áreas vegetadas e sua idade, seja obtido cada vez mais de forma automática, devido à dificuldade de amplas investigações em campo, que torna o processo moroso, sem contar a dificuldade e limitação em acessar diversas áreas, portanto é importante a exploração de técnicas avançadas de análise de imagens para desenvolver sistemas automatizados que tragam eficiência em seus resultados.

\section{CONCLUSÕES}

A maioria dos pesquisadores utilizou imagens Landsat para identificar os estágios sucessionais e recuperar a idade da vegetação, devido à disponibilidade dos dados numa série histórica de mais de 40 anos, todavia pesquisas onde a idade era conhecida utilizaram-se de combinações e outros tipos de dados, para melhorar a capacidade de discriminação da vegetação.

Os métodos empregados envolveram técnicas de classificação e modelos preditivos: Pósclassificação de fusão, Classificação Supervisionada, Feição de textura, Índices Espectrais, Modelo de Mistura Espectral (MME), Modelo de Regressão, Redes Neurais (RN), Árvores de Decisão e Floresta Aleatória. Destacando o uso das técnicas não-paramétricas em 6 (seis) estudos, refinando o processo de identificação da idade da vegetação em relação às classificações tradicionais. Isto se torna relevante, uma vez que os pesquisadores estão buscando metodologias diferenciadas para obter o mapeamento dos estágios sucessionais e sua idade, diversificando os métodos de aplicação.

Foi possível entender com a revisão de literatura a importância da floresta secundária e de que a idade da vegetação é uma variável fundamental para compreender a função da vegetação; permitir o acompanhamento do processo de regeneração e recuperação de áreas; auxiliar na avaliação e quantificação do carbono; e determinar a qualidade ecossistêmica do sítio, entre outros. Entretanto ficou evidente que não é possível a medição direta da idade da vegetação, mas sim, as manifestações que são sua consequência, sendo estas únicas para cada localidade, dependendo do histórico e da trajetória de distúrbio, sendo o que foi constatado com este trabalho, já que cada pesquisador encontrou uma estimativa de idade de acordo com a área de estudo, trajetória do distúrbio, processo de regeneração e devido os dados e técnicas de SR empregados.

Conclui-se a importância do uso do SR nos estudos analisados, entretanto ressalta-se que ainda existem dificuldades na classificação automática devido à similaridade espectral entre os 
estágios sucessionais a partir de uma determinada idade, sendo mais fácil a sua detecção em períodos mais recentes ao distúrbio ocorrido. Contudo, informações espectrais e texturais, qualidade dos produtos do SR e os distintos métodos de classificação e predição estão fornecendo respostas cada vez mais confiáveis, mesmo que em áreas de estudos específicas e pontuais.

\section{REFERÊNCIAS BIBLIOGRÁFICAS}

ALVES, D. S.; SKOLE, D. L. Characterizing land cover dynamics using multitemporal imagery. International Journal of Remote Sensing. 17(4), p. 835-839, 1996.

AMARAL, M. V. F; LOPES, A. L.; SOARES, V. P.; BOECHAT, C. P. S.; LEITE, H. G.; MARTINS, S. V.; FERNANDES FILHO, E. I.; LANA, J. M. Avaliação e comparação de métodos de classificação de imagens de satélites para o mapeamento de estádios de sucessão florestal. Revista Árvore, 33, p. 575-82, 2009.

ATTANASIO, C. M.; RODRIGUES, R. R.; GANDOLFI, S.; NAVE, A. G. Adequação ambiental de propriedades rurais: recuperação de áreas degradadas e restauração de matas ciliares. Piracicaba: ESALQ, Depto. Ciências Florestais, 66 p., 2006. Relatório técnico.

BREIMAN, L. Random forests. Machine Learning, 2001, 45: p. 5-32.

BRONDIZIO, E.; MORAN, E.; MAUSEL, P.; WU; Y. Land cover in the Amazon estuary: Linking of the Thematic Mapper with botanical and historical data. Photogrammetric Engineering and Remote Sensing, 62(8): p. 921-929, 1996.

CARREIRAS, J. M. B.; JONES, J.; LUCAS, R. M.; SHIMABUKURO, Y. E. Mapping major land cover types and retrieving the age of secondary forests in the Brazilian Amazon by combining single-date optical and radar remote sensing data. Remote Sensing of Environment, v. 194, p. 16-32, 2017.

CHAZDON, R. L.; PERES, C. A.; DENT, D.; SHEIL, D.; LUGO, A. E.; LAMB, D.; STORK, N. E.; MILLER, S. E. The potential for species conservation in tropical secondary forests. Conservation Biology 23(6): p. 1406-1417, 2009.

COHEN, W.B.; SPIES, T.A.; FIORELLA, M. Estimating the age and structure of forests in a multi-ownership landscape of western Oregon, U.S.A. International Journal of Remote Sensing, 16(4): p. 721-746, 1995.

DENT, D. H.; WRIGHT, S. J. The future of tropical species in secondary forests: a quantitative review. Biological Conservation 142: p. 2833-2843, 2009.

DENYER, D.; TRANFIELD, D. Producing a systematic review. In D. A. Buchanan \& A. Bryman (Eds.), The SAGE handbook of organizational research methods. London: Sage Publications Ltda, 2009, p. 671-689.

DIXON, R. K.; BROWN, S.; HOUGHTON, R. A.; SOLOMON, A. M.; TREXLER, M. C.; WISNIEWSKI, J., Carbon pools and flux of global forest ecosystems, Science, 263, 185- 190, 1994.

FEARNSIDE, P.M. AND W.M. GUIMARÃES. Carbon uptake by secondary forests in Brazilian Amazonia. Forest Ecology and Management 80(1-3): 35-46, 1996.

FOODY, G.M.; PALUBINSKAS, G.; LUCAS, R.M.; CURRAN, P.J.; HONZAK, M. Identifying terrestrial carbon sinks: classification of successional stages in regenerating tropical forest from Landsat TM data. Remote Sensing of Environment, 55: p. 205-216, 1996.

FOSBERG, F.R. Succession andcondiction of ecossystems. The Journal of the Indian Botanical Society. XLVI (4): p. 312316, 1967.

FRASER, R.H.; LI, Z. Estimating fire-related parameters in boreal forest using SPOT VEGETATION, Remote Sensing of Environment, 82: p. 95-110, 2002. 
FRIEDL, M. A.; BRODLEY, C. E. Decision Tree Classification of Land Cover from Remotely Sensed Data. Remote Sensing of Environment, 61 (3): p.399-409, 1997.

GALVÃO, L. S.; PONZONI F. J.; LIESENBERG, V.; SANTOS, J. R. Possibilities of Discriminating Tropical Secondary Succession in Amazônia using Hyperspectral and Multiangular CHRIS/PROBA Data. International Journal of Applied Earth Observation and Geoinformation, 11(1), p. 8-14, 2009.

GARZA-REYES, J.A. Green lean and the need for Six Sigma. Int. J. Lean Six Sigma 6, p. 226-248, 2015.

GOMES, M. F.; MAILLARD, P. O uso de feições de textura em imagens RapidEye para estimativas da idade e de parâmetros estruturais da vegetação do cerrado. Anais XVI Simpósio Brasileiro de Sensoriamento Remoto - SBSR, Foz do Iguaçu, PR, Brasil, 13 a 18 de abril de 2013, INPE, v. 1, p. 3260-3267, 2013.

GRANT, L. Diffuse and specular characteristics of leaf reflectance. Remote Sensing of Environment , 22, p.309-322, 1987.

HANSEN, M.; DUBAYAH, R.; DEFRIES, R. Classification trees: An alternative to traditional land cover classifiers. International Journal of Remote Sensing, 17(5): p. 1075-1081, 1996.

HELLER, R. C.; ULLIMAN, J. J. Manual of Remote Sensing, v.2 (2nd ed.)., Chapter Forest resource assessments. Falls Church: American Society of Photogrammetry. p. 2229-2324, 1983.

HELMER, EILEEN H.; LEFSKY, MICHAEL A.; ROBERTS, D. A. Biomass accumulation rates of Amazonian secondary forest and biomass of old-growth forests from Landsat time series and the Geoscience Laser Altimeter System. Journal of Applied Remote Sensing, v. 3, n. 1, p. 033505, 2009.

HELMER, E. H.; BROWN, S.; COHEN, W. B. Mapping montane tropical forest successional stage and land use with multi-date Landsat imagery. International Journal of Remote Sensing, v. 21, n. 11, p. 2163-2183, 2000.

KAGEYAMA, P. Y.; CASTRO, C. F. A. Sucessão secundária, estrutura genética e plantações de espécies arbóreas nativas. IPEF, Piracicaba, n.41/42, p.83-93, 1989.

KENNAWAY, T.; HELMER, E. H. The Forest Types and Ages Cleared for Land Development in Puerto Rico. GIScience \& Remote Sensing, v. 44, n. 4, p. 356-382, 2007.

KIMES, D. S.; NELSON, R. F.; SALAS, W. A.; SKOLE, D. L. Mapping secondary tropical forest and forest age from SPOT HRV data. International Journal of Remote Sensing, v. 20, n. 18, p. 3625-3640, 1999.

KLEIN, R.M. Ecologia da Flora e Vegetação do Vale do Itajaí. Sellowia, v.32, p.164-369, 1980.

KRISHNASWAMY, J.; KIRAN, M.C.; GANESHAIAH, K. N. Tree model based eco- climatic vegetation classification and fuzzy mapping in diverse tropical deciduous ecosystems using multi-season NDVI, International Journal of Remote Sensing, 25(6): p. 1185-1205, 2004.

JENSEN, R.; QIU, FANG; JI, MINHE, J. Predictive modelling of coniferous forest age using statistical and artificial neural network approaches applied to remote sensor data. International Journal of Remote Sensing, v. 20, n. 14, p. 28052822, 1999.

LAWRENCE, R.L.; WRIGHT, A. Rule-based classification systems using classification and regression tree (CART) analysis, Photogrammetric Engineering and Remote Sensing, 67(10): p.1137-1142, 2001.

LI, D.; JU, W.; FAN, W.; GU, Z. Estimating the age of deciduous forests in northeast China with Enhanced Thematic Mapper Plus data acquired in different phenological seasons. Journal of Applied Remote Sensing, v. 8, n. 1, p. 083670, 2014.

LI, G.; LU, D.; MORAN, E. F.; HETRICK, S. Land-Cover Classification in a Moist Tropical Regionof Brazil with Landsat Thematic Mapper Imagery. International Journal of Remote Sensing, 32(23), p. 8207-8230, 2011.

LIU, W.T.H., Aplicações de Sensoriamento Remoto. Campo Grande: Editora UNIDERP. 2006, 908 p. 
LU, D.; MORAN, E.; BATISTELLA, M. Linear mixture model applied to Amazonian vegetation classification. Remote Sensing of Environment, v. 87, n. 4, p. 456-469, 2003.

LU, D.; BATISTELLA, M.; LI, G.; MORAN, E.; HETRICK, S.; FREITAS, C. C.; DUTRA, L. V; SANT'ANNA, S. J. S. Land use/Cover Classification in the Brazilian Amazon using Satellite Images. Brazilian Journal of Agricultural Research, 47 (9), p. 1185-1208, 2012.

LU, D.; LI, G.; MORAN, E.; KUANG, W. A comparative analysisof approaches for successional vegetation classification in the Brazilian Amazon. GIScience \& Remote Sensing, 51(6), p. 695-709, 2014.

LUCAS, R. M.; HONZAK, M.; FOODY,G. M.; CURRAN, P. J.; CORVES, C. Characterizing tropical secondary forests using multi-temporal Landsat sensor imagery, International Journal of Remote Sensing. 14, p.3061-3067, 1993.

LUCAS, R. M.; HONZÁK, M.; CURRAN, P. J.; FOODY, G. M.; MILNE, R.; BROWN, T.; AMARAL, S. Mapping the regional extent of tropical forest regeneration stages in the Brazilian Legal Amazon using NOAA AVHRR data. International Journal of Remote Sensing, v. 21, n. 15, p. 2855-2881, 2000.

MAILLARD, P.; COSTA-PEREIRA, P. S. Estimating the age of cerrado regeneration using Landsat TM data. Canadian Journal of Remote Sensing, v. 36, n. S2, p. 243-256, 2010.

MEDEIROS, J.F.; RIBEIRO, J.L.D.; Cortimiglia, M.N., Success factors for environmentally sustainable product innovation: In: A systematic literature review. J. Clean. Prod. 65, p. 76-86, 2014.

MORAN, E.F., BRONDÍZIO, E.S., TUCKER, J.M., DA SILVA-FORSBERG, M.C., FALESI, I., MCCRACKEN, S.D. Strategies for Amazonian forest restoration: evidence for afforestation infive regions of the Brazilian Amazon. In: Hall, A. (Ed.), Amazônia at the Crossroads: The Challenge of Sustainable Development.Institute for Latin American Studies, University of London, London, pp. 129-149. 2000.

NEEFF, T.; LUCAS, R. M.; SANTOS, J. R. DOS; BRONDIZIO, E. S.; FREITAS, C. C. Area and age of secondary forests in Brazilian Amazonia 1978-2002: An empirical estimate. Ecosystems, v. 9, n. 4, p. 609-623, 2006.

NUNES, S. S. S. Estimativas de biomassa e carbono e indicadores para restauração de florestas secundárias em Paragominas - Pará / Sâmia do Socorro Serra Nunes. Piracicaba, 2011. 126 p.

PAL, M.; MATHER P.M. An assessment of the effectiveness of decision tree metods for land cover classification, Remote Sensing of Environment, 86: p. 554-565, 2003.

PETERSON, D. L.; ABER, J. D.; MATSON, P. A.; CARD, D. H.; SWANBERC, N.; WESSAN, C.; SPANNER, M. Remote sensing of forest canopy and leaf biochemical contents. Remote Sensing of Environment, 24, p.85 $\pm 108,1988$.

PONZONI, F. J. Comportamento Espectral da Vegetação. In: MENESES, P. R., NETTO, J. S. M. (org) Sensoriamento remoto, refletância dos alvos naturais. Brasília - DF: Editora Universidade de Brasília - UNB, Embrapa Cerrados, p.157-199, 2001.

QUINTANO, C.; FÉRNANDEZ-MANSO, A.; SHIMABUKURO, Y. E.; PEREIRA, G. Aplicação do modelo linear de mistura espectral para o mapeamento de queimadas no Parque Nacional das Emas. In: Anais XV Simpósio Brasileiro de Sensoriamento Remoto - SBSR, Curitiba, PR, Brasil, 30 de abril a 05 de maio de 2011, INPE p.7910.

ROGAN, J.; MILLER, J.; STOW, D.; FRANKLIN, J.; LEVIEN, L.; FISCHER, C. Land-cover change monitoring with classification treesusing Landsat TM and ancillary data, Photogrammetric Engineering and Remote Sensing, 69: $p$. 793-804, 2003.

SHIMABUKURO, Y. E.; SMITH, J. A. The least-square mixing models to generate fraction images derived from remote sensing multispectral data. IEEE Transactions on Geoscience and Remote Sensing, v. 29, n. 1, p. 16- 20, 1991.

SKIDMORE, A. K. An Expert System Classifies Eucalypt Forest Types Using Thematic Mapper Data and a Digital Terrain Model, Photogrammetric Engineering and Remote Sensing, 55: p.1449-1464, 1989. 
STEININGER, M. K. Tropical secondary forest regrowth in the Amazon: age, area and change estimation with Thematic Mapper data. International Journal of Remote Sensing, 17, p. 9-27, 1996.

STRAHLER, A. H. Stratification of Natural Vegetation for Forest and Rangeland Inventory Using Landsat Imagery and Collateral Data, International Journal of Remote Sensing, 2: p.15-41, 1981.

TRABAQUINI, K.; MIGLIORANZA, E.; FRANÇA, V.; VIEIRA, A. O. S. Análise espacial de fragmentos florestais com ocorrência de Jaracatiá. Revista RA'E GA - O Espaço Geográfico em Análise. Vol. 17, pág. 193 - 203, 2007.

UHL, C.; BUSCHBACKHER, R.; SERRÃO, A. S. Abandoned pastures in eastern Amazonia I. patterns of plant succession, J. Ecol. 75, 663-681, 1988.

VieirA, I. C.G.; ALMEIDA, A. S.; DAVIDSON, E. A.; StOne, T. A.; CARVAlHO, C. J.; GUerRero, J. B. Classifying successional forest using Landsat spectral properties and ecological characteristics in eastern Amazônia. Remote Sensing of Environment, v. 87, p.470-481, 2003.

WOODCOCK, C. E.; MACOMBER, S. A.; PAX-LENNEY, M.; COHEN, W. B. Monitoring Large Areas for Forest ChangeUsing Landsat: Generalization across Space, Time, and Landsat Sensors. Remote Sensing of Environment, 78(1-2): p.194203, 2001.

WULDER, M. Optical remote-sensing techniques for the assessment of forest inventory and biophysical parameters. Progress in Physical Geography, v.22, n. 4, p. 449-476, 1998. 